\title{
Erratum: Finite measure for the initial conditions of inflation [Phys. Rev. D 99, 063515 (2019)]
}

\author{
Kieran Finn and Sotirios Karamitsos
}

(2) (Received 3 May 2019; published 24 May 2019)

DOI: 10.1103/PhysRevD.99.109901

During a review of the code used to obtain (48), a mistake was discovered. The change of variables in (42)-(44) was not correctly implemented, and thus, Eqs. (48)-(50) are incorrect. As a result, the numerical results in Figs. 1 and 2 are also incorrect. The correct calculation and updated plots follow.

In order to simplify the maths, we will alter the definitions in (42)-(44) to read

$$
\begin{gathered}
H \equiv \frac{\rho}{\sqrt{6}} \cos \alpha, \\
\dot{\varphi} \equiv \rho \sin \alpha \cos \beta, \\
H_{\chi} \equiv \rho \sqrt{V} \sin \alpha \sin \beta .
\end{gathered}
$$

These definitions still have the desired property that $\rho$ is the only coordinate affected by time dilations. Despite this alteration, Eqs. (54) and (55) in the original publication are unchanged.

The Hamiltonian expressed in terms of the coordinate chart $\Phi^{\alpha}=\{\lambda, \varphi, \tilde{\chi}, \rho, \alpha, \beta\}$ is

$$
\mathcal{H}=\mathcal{L}=-\frac{1}{2} e^{3 \lambda} \rho^{2} \cos (2 \alpha)
$$

Thus, the Hamiltonian constraint (31) becomes simply

$$
\alpha=\frac{\pi}{4}
$$
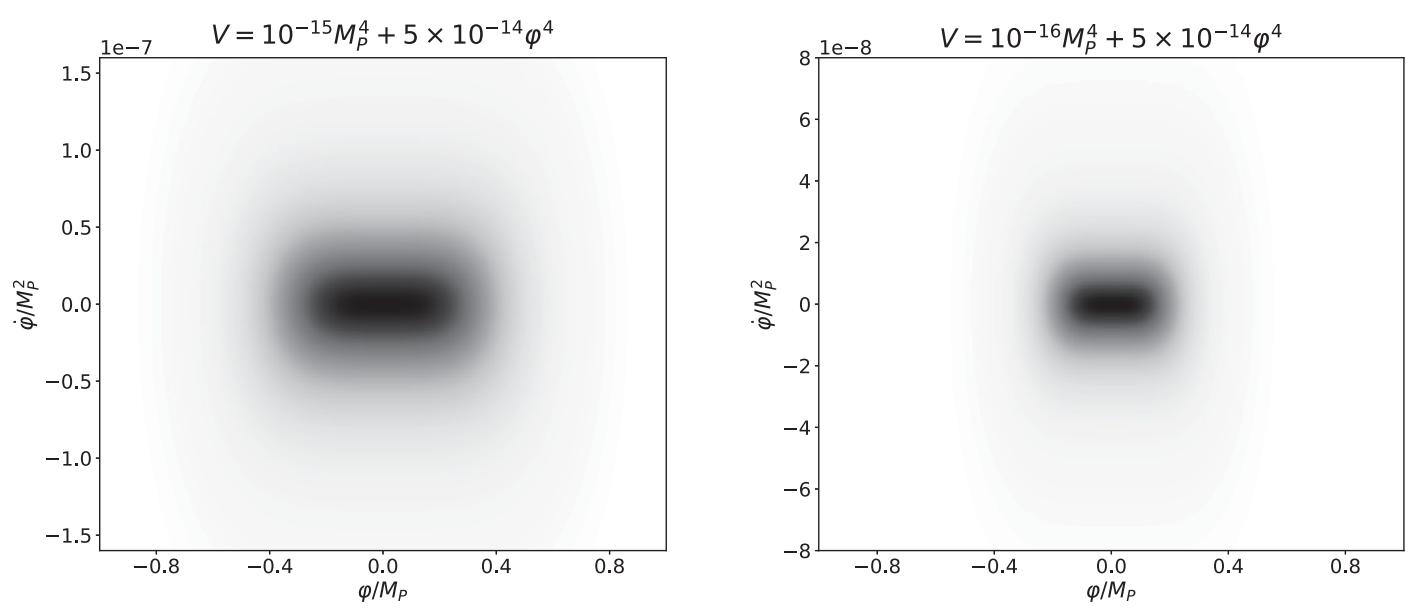

FIG. 1. Distribution of the values of $\varphi$ and $\dot{\varphi}$ on the phase-space manifold for the potential $V(\varphi)=\lambda \varphi^{4}+\Lambda$. Darker areas correspond to a higher value of $P$ as given, defined in (9). We have fixed $\lambda=5 \times 10^{-14}$ for both plots by the amplitude of scalar perturbations in the CMB. The left and right plots correspond to $\Lambda=10^{-15}$ and $\Lambda=10^{-16}$, respectively. Notice how reducing the value of $\Lambda$ causes the distribution to become more tightly clustered around $\varphi=0$. 


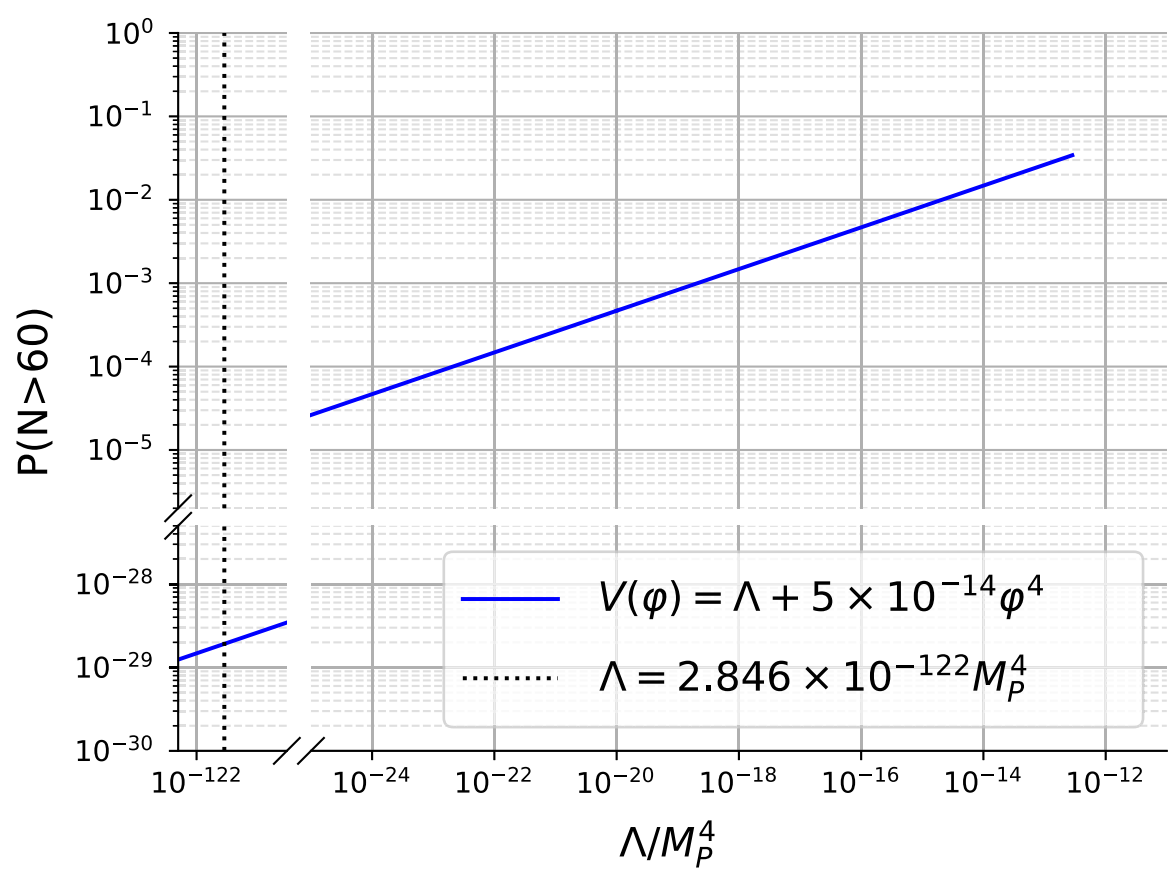

FIG. 2. Fraction of phase space that allows $N>60 e$-foldings of inflation as a function of the cosmological constant $\Lambda$ for an inflationary potential $V(\varphi)=\lambda \varphi^{4}+\Lambda$. The amplitude of scalar perturbations in the CMB has been used to set $\lambda=5 \times 10^{-14}$. A vertical line is placed at $\Lambda=2.846 \times 10^{-122} M_{P}^{4}$, corresponding to the observed value of the cosmological constant today.

In order to study only physically allowed sets of initial conditions, we must restrict ourselves to the five-dimensional hypersurface on which (5) is satisfied (the Hamiltonian hypersurface). The induced metric on this hypersurface can be calculated from (23) in the original publication. However, we find it to be singular, with

$$
\tilde{\mathcal{G}}_{\alpha 4}=\tilde{\mathcal{G}}_{4 \alpha}=0
$$

for all values of the phase space index $\alpha$. Here 4 corresponds to the $\rho$ coordinate. As a consequence, the invariant volume element of this submanifold vanishes. Therefore, we cannot use it in order to construct a measure to help us distinguish between finely tuned and generic sets of initial conditions.

To remedy the above problem, we use a regularization technique. We look at a hypersurface very close to the Hamiltonian hypersurface, where

$$
\mathcal{H}=\epsilon,
$$

and take the limit as $\epsilon \rightarrow 0$. The invariant volume element on this perturbed Hamiltonian hypersurface is

$$
d \tilde{\Omega}(\epsilon)=\sqrt{\frac{6 e^{15 \lambda} \rho^{2} \epsilon}{e^{3 \lambda} \rho^{2}-2 \epsilon}} \frac{1}{\sqrt{V}} d \lambda d \varphi d \tilde{\chi} d \rho d \beta .
$$

Notice that the total volume of the hypersurface is proportional to $\sqrt{\epsilon}$ and vanishes as $\epsilon$ tends to zero. However, as we will see, this dependence on $\epsilon$ will drop out of any ratio of physically distinguishable sets of initial conditions.

We remind the reader that the coordinates $\lambda, \tilde{\chi}$, and $\rho$ are all physically irrelevant and must be integrated out. This leads to the following distribution:

$$
\begin{aligned}
P\left(\varphi_{1}, \varphi_{2}, \beta_{1}, \beta_{2}\right) & =\lim _{\epsilon \rightarrow 0} \lim _{\Lambda_{\lambda}, \Lambda_{\tilde{\chi}} \Lambda_{\rho} \rightarrow \infty} \frac{\int_{\tilde{\chi}=-\Lambda_{\tilde{\chi}}}^{\tilde{\chi}=\Lambda_{\tilde{\chi}}} \int_{\lambda=-\Lambda_{\lambda}}^{\lambda=\Lambda_{\lambda}} \int_{\rho=0}^{\rho=\Lambda_{\rho}} \int_{\varphi=\varphi_{1}}^{\varphi=\varphi_{2}} \int_{\beta=\beta_{1}}^{\beta=\beta_{2}} d \tilde{\Omega}(\epsilon)}{\int_{\tilde{\chi}=-\Lambda_{\tilde{\chi}}}^{\tilde{\chi}=\Lambda_{\tilde{\chi}}} \int_{\lambda=-\Lambda_{\lambda}}^{\lambda=\Lambda_{\lambda}} \int_{\rho=0}^{\rho=\Lambda_{\rho}} \int_{\varphi=-\infty}^{\varphi=\infty} \int_{\beta=0}^{\beta=2 \pi} d \tilde{\Omega}(\epsilon)} \\
& =\frac{\beta_{2}-\beta_{1}}{2 \pi \mathcal{N}} \int_{\varphi=\varphi_{1}}^{\varphi=\varphi_{2}} \frac{1}{\sqrt{V(\varphi)}} d \varphi,
\end{aligned}
$$


which replaces Eq. (53) in the original publication. Here, $P\left(\varphi_{1}, \varphi_{2}, \beta_{1}, \beta_{2}\right)$ is the fraction of phase space for which $\varphi_{1}<\varphi<\varphi_{2}$ and $\beta_{1}<\beta<\beta_{2}$, and

$$
\mathcal{N}=\int_{-\infty}^{\infty} \frac{1}{\sqrt{V(\varphi)}} d \varphi
$$

is a normalization constant.

The distribution (9) is well-defined, provided $\mathcal{N}$ is finite. This in turn requires that $V(\varphi)$ is strictly greater than 0 and that it approaches infinity faster than $\varphi^{2}$ as $\varphi \rightarrow \infty$. This condition is stronger than the one originally published. In particular, it means that the common inflationary potential $V(\varphi)=\frac{1}{2} m^{2} \varphi^{2}+\Lambda$ can no longer be used as an example. Instead, we concentrate on the quartic potential $V(\varphi)=\lambda \varphi^{4}+\Lambda$. The distribution of the field and its derivative for such a potential is shown in Fig. 1. The fraction of phase space allowing for $N>60 e$-foldings of inflation is shown as a function of the cosmological constant $\Lambda$ in Fig. 2. These figures replace those of the original publication. 